\title{
BRCA2 gene mutation, c.2808_2811delACAA (p.Ala938Profs), in male breast cancer - clinicopathological analysis based on a case report
}

\author{
Joanna Huszno ${ }^{1}$, Anna Fiszer-Kierzkowska², Wojciech Pigłowski², Magdalena Mazur ${ }^{2}$ \\ ${ }^{1}$ Clinical and Experimental Oncology Department, Maria Skłodowska-Curie Memorial Cancer Center and Institute of Oncology, \\ Gliwice Branch, Gliwice, Poland \\ ${ }^{2}$ Center for Translational Research and Molecular Biology of Cancer, Maria Skłodowska-Curie Memorial Cancer Center and Institute \\ of Oncology, Gliwice Branch, Gliwice, Poland
}

\begin{abstract}
Male breast cancer $(\mathrm{MBC})$ is a rare disease that occurs in $\sim 0.2 \%$ of all neoplasms among men. The risk of developing $\mathrm{MBC}$ is higher in men with a $B R C A 2$ genetic mutation (7\%). The aim of this study was to evaluate the association between c.2808_2811del ACAA (p.Ala938Profs) BRCA2 mutation in MBC and clinicopathological factors. A 75-year-old patient was admitted to the Genetic Outpatient Clinic with a diagnosis of right breast cancer and with a family history of cancer (two daughters who were diagnosed with breast cancer at ages 46 and 38 years). Postoperative histopathological examination revealed tumor type pT2 N1a Mx, NST NG-3 G-3, Ki-67 (75\%), HER2 (+), ER (+++), PR (+++), invasive carcinoma and luminal B subtype. The complete coding sequences of the BRCA1 and $B R C A 2$ genes were analyzed for genomic DNA material using next generation sequencing on the lon Torrent platform. The c.2808_2811delACAA (p.Ala938Profs) mutation was observed in the BRCA2 gene. The presence of the c.2808_2811delACAA (p.Ala938Profs) mutation in the BRCA2 gene was confirmed using the Sanger method. The same $B R C A 2$ gene mutation was reported in one of the patient's daughters. The detected mutation causes the frameshift mutation, resulting in the creation of an additional translation termination codon and the synthesis of an abnormal protein. This mutation was associated with a later age of disease, a higher histological degree, a higher mitotic index, a positive steroid receptor status and the luminal B subtype HER2- breast cancer.
\end{abstract}

Key words: BRCA2 gene mutation, male breast cancer, NGS, clinicopathological factors.

\section{Introduction}

Male breast cancer (MBC) is a rare disease that occurs in $\sim 0.2 \%$ of all neoplasms among men. There are currently only single case reports in the literature that describe the diagnostics and treatment of male breast cancer [1-5]. The European Organization for Research and Treatment of Cancer (EORTC), Translational Breast Cancer Research Consortium (10085/TBCRC) / Breast International Group (BIG) / North American Breast Cancer Groups (NABCG) International Male Breast Cancer Programme revealed that $M B C$ presents usually as luminal A like subtype, $88 \%$ of cases express androgen receptors, only $1 \%$ are triple negative, and $9 \%$ are HER-2 positive [6]. Males that harbor the BRCA1 gene mutation have a higher risk of developing breast cancer (1\%). The risk of male breast cancer is also higher in males with the BRCA2 gene mutation (7\%) [7]. Cases of male breast cancer are more common in BRCA2 than BRCA1 families. Prostate cancer is a recognized component of the hereditary breast and ovarian cancer (HBOC) syndrome tumor spectrum, where inherited susceptibility is accounted for by mutations in BRCA1 and BRCA2. The overall risk of prostate cancer has been reported as up to 3.8-fold for men who carry BRCA1 mutations, and up to 8.6-fold for men who carry BRCA2 mutations. Mutations in BRCA2 have been associated with more aggressive clinicopathological characteristics of prostate cancer and worse outcomes.

The predominant histological subtype (> 80\%) of male breast cancer is invasive ductal carcinoma. Lobular carcinoma is rare [7]. Histopathological features of male breast cancer are: intermediate or high histological grade (G2-G3), estrogen receptor (ER) positive (90\%), progesterone receptor (PR) positive (80-96\%) and human epidermal growth factor receptor 2 (HER2) negative $(\sim 15 \%$ of MBCs exhibited upregulated HER2 expression), expression of androgen receptors (range 39-95\%) [8-10]. Breast cancer types in males are pri-
Corresponding author:

Joanna Huszno MD, Clinical and Experimental Oncology Department, Maria Skłodowska-Curie Memorial Cancer Center and Institute of Oncology, Gliwice Branch, 15 Wybrzeże Armii Krajowej St., 44-101 Gliwice, Poland, e-mail: joahus@wp.pl
Submitted: 5.08 .2019

Accepted: 25.11 .2019 
marily invasive ductal carcinoma with higher stage and lower grade, and are more often ER+ and HER2subtypes in comparison with woman with breast cancer. $M B C s$ exhibit worse prognosis than female breast cancer due to the often advanced stage at diagnosis, higher age and frequent presence of comorbidities [11].

There are three founder alleles of BRCA1 (c.181T $>\mathrm{G}$, c.4034delA and c.5266dupC) that were reported in Poland in 2000, and these three mutations have comprised the standard testing panel used throughout the country. Since that time, new original mutations (c.68_69delAG, c.676del, c.3700_3704delGTAAA, c.3756_3759delGTCT and $c .5251 C>T$ ) have been described. Certain recurrent mutations of $B R C A 2$ have also been reported (c.658_659delGT, c.3847_3848delGT, c.5239_5240insT, c.5946delT and c.7913_7917del) in Polish families [12].

According to the BIG database, c.2808_2811del ACAA p. (p.Ala938Profs) is the second most reported frameshift BRCA2 mutation and the most prevalent mutation in Spain [13]. This mutation has been reported in almost all Western European countries [14] and in African women with premenopausal breast cancer [15]. The detected mutation has already been described twice in the Polish population, and is also the third most common pathogenic mutation in BRCA2 in the Caucasian population. It is believed that the high frequency of this mutation is associated with its location in the "hot spot of the mutation" (mutational hot spot) [16]. The coexistence of the c.2808_2811delACAA mutation with MBC has already been described [17].

The present paper reports the case of a 75-year-old man who was diagnosed with a locally advanced invasive ductal carcinoma and with family history of breast cancer (two daughters with breast cancer aged 46 and 38 years). The aim of the present study was to evaluate the presence of c.2808_2811del ACAA (p.Ala938Profs) $B R C A 2$ mutation in $M B \bar{C}$ and to assess the association between the aforementioned mutation and clinicopathological factors.

\section{Case report}

A 75-year-old patient was admitted to the Genetic Outpatient Clinic in Maria Skłodowska-Curie Memorial Cancer Center and Institute of Oncology, Gliwice Branch, with a diagnosis of right breast cancer and with a family history of cancer (two daughters who were diagnosed with breast cancer at ages 46 and 38 years). In the physical examination, a tumor was detected in the central area of the right breast that was $4 \mathrm{~cm} \times 4 \mathrm{~cm}$ in size, engaging the nipple and infiltrating its base. In the right axillary plane, a lymph node was examined with dimensions of $1.5 \mathrm{~cm} \times 2.0 \mathrm{~cm}$. Other regional lymph nodes were not enlarged. On 11 January, 2018, PET testing was performed. The PET/CT scan demonstrated the presence of right breast cancer (tumor sized
$28 \mathrm{~mm} \times 23 \mathrm{~mm}$ ) with metastasis in the right armpit (lymph node sized $10 \mathrm{~mm} \times 5 \mathrm{~mm}$ ). A thick-needle biopsy was performed. Histopathological examination revealed ductal invasive carcinoma. On 19 February, 2018, a radical amputation of the right breast with lymphadenectomy was performed. Postoperative histopathological examination demonstrated a tumor with the presence of angioinvasion, without neuroinvasion, pT2 N1a Mx, NST NG-3 G-3, Ki-67 75\%, ER (+++), PR (+++), and without upregulated HER2 expression. Metastases were demonstrated in two of 40 lymph nodes. The metastatic lymph nodes were up to $0.5 \mathrm{~cm}$ in diameter with transgression of the nodal sac.

The patient was qualified for adjuvant chemotherapy with $A C$ regimens, and then for radiotherapy and hormonotherapy based on aromatase inhibitors. Genetic counseling was also performed. During the genetic consultation, a family history was collected, the pedigree was removed and the medical records were reviewed. The patient provided written informed consent regarding the use of his biological material for clinical research (all were routine laboratory analyses). The complete coding sequence of the BRCA1 and BRCA2 genes was analyzed on the genomic DNA material using next generation sequencing (NGS) on the Ion Torrent platform. The c.2808_2811delACAA p. (Ala938Profs) mutation was observed in the BRCA2 gene. The presence of the c.2808_2811delACAA p. (Ala938Profs) mutation in the $B R C A 2$ gene was confirmed using the Sanger method. The detected mutation causes a frameshift mutation, resulting in the creation of an additional translation termination codon and the synthesis of an abnormal protein. Genetic consultation with subsequent genetic testing was recommended for adult family members. Cancer was present in the patient's family history, with his two daughters both having developed breast cancer. One daughter was diagnosed aged 41 in 2010. She received radical amputation of the right breast with lymphadenectomy. Histopathological examination revealed ductal invasive carcinoma, pT2pN2, ER (++), PR (+++) and HER2 that was not upregulated. She was qualified for adjuvant chemotherapy with FAC regimens, and then for radiotherapy and hormonotherapy based on tamoxifen. Genetic counseling was also performed. The tested material had genomic DNA isolated from peripheral venous blood. No c.68_69delAG, c.181T>G, c.4034delA, c.5266dupC or c.3700_3704del5 mutations were detected in the BRCA1 gene (reference sequence number GenBank NM_007294.3), or c.5946delT and c.9403delC in the BRCA2 gene. Test methods included restriction fragment length polymorphism-PCR for mutations in c.181T>G (BRCA1) and c.9403delC (BRCA2); and allele-specific amplification PCR for other mutations (BRCA1). The second daughter was diagnosed aged 38 years in 2014. She had left breast cancer type cT4bN3M0. A thick-needle biopsy was performed. His- 
topathological examination revealed lobular invasive carcinoma G2, ER (+), PR (+), HER2 that was not upregulated. She had neoadjuvant chemotherapy with AC and paclitaxel regimens, and then hormonotherapy based on tamoxifen. On 8 August, 2014, numerous metastases in the CNS were detected in the CT scan of the head. She received radiotherapy, and genetic counseling was also performed. No c.68_69delAG, c.181T>G, c.4034delA, c.5266dupC and c.3700_3704del5 mutations were observed in the BRCA1 gene (reference sequence number GenBank NM_007294.3), or c.5946delT and c.9403delC in the BRCA2 gene. There were also no mutations observed in the CHEK2 gene (c.1100delC and mutation c.470T>C) (GenBank NM_145862.2.). However, the presence of the c.2808_2811delACAA (p.Ala938Profs) mutation in the BRCA2 gene was confirmed using the Sanger method; the same mutation was reported in her father.

\section{Conclusions}

The presence of the c.2808_2811delACAA (p.Ala938Profs) mutation in the BRCA2 gene was associated with a diagnosis at a later age, a higher histological degree, a higher mitotic index (Ki-67), a positive steroid receptor status, the luminal B subtype and HER2- breast cancer. This mutation is also associated with higher predisposition to hereditary breast cancer.

NGS improved the detection rates of mutations in Polish patients with familial breast cancer. NGS-based testing should be performed in patients with breast or ovarian cancer who present with $B R C A 1 / 2$ negative tests for the selected $B R C A 1 / 2$ pathogenic mutations.

\section{Disclosure}

The authors report no conflict of interest.

\section{References}

1. Pietrzyński Ł, Rajcher M, Pietrzyńska T. Leczenie raka piersi u mężczyzn - opis przypadku. Treatment of male breast cancer - case report. Med Paliat 2017; 9: 176-180.

2. Park SY, Cho YU, Kim SJ, Hur MH. A case report of 29-year-old male patient with breast carcinoma. Korean J Clin Oncol 2017; 13: 50-53.

3. O'Leary TR, Shriver CD, Wind G. Metachronous Contralateral Male Breast Cancer: Case Report and Literature Review. Mil Med. 2019; 184: e581-e586.

4. Wroński K. Male breast cancer - case report and review of literature. Borgis - New Medicine 2014; 2: 52-54.

5. Mishra T, Kelkar R, Navare M, et al. Male Breast Carcinoma Case Report and Review of Literature. J Med Sci Clin Res 2018; 6: 478-483.

6. Ahmed M, Esposito E. Report from the 37th San Antonio breast cancer symposium, 9-13th December 2014, Texas, USA. Ecancermedicalscience 2015; 9: 508.

7. Fentiman IS, Fourquet A, Hortobagyi GN. BRCA1 and BRCA2 for men. Information for men from families with a known alteration in the BRCA1/2 gene. Male breast cancer. Lancet 2006; 367: 595-604.
8. Giordano SH, Cohen DS, Buzdar AU, et al. Breast carcinoma in men: a population-based study. Cancer 2004; 101: 51-57.

9. Muñoz de Toro MM, Maffini MV, Kass L, Luque EH. Proliferative activity and steroid hormone receptor status in male breast carcinoma. J Steroid Biochem Mol Biol 1998; 67: 333-339.

10. Ge Y, Sneige N, Eltorky MA, et al. Immunohistochemical characterization of subtypes of male breast carcinoma. Breast Cancer Res 2009; 11: R28.

11. Ottini L, Capalbo C, Rizzolo P, et al. HER2-positive male breast cancer: an update. Breast Cancer (Dove Med Press) 2010; 2: 45-58.

12. Cybulski C, Lubiński J, Wokołorczyk D, et al. Mutations predisposing to breast cancer in 12 candidate genes in breast cancer patients from Poland. Clin Genet 2015; 88: 366-370.

13. Diez O, Gutiérrez-Enríquez S, Balmaña J. Heterogeneous prevalence of recurrent BRCA1 and BRCA2 mutations in Spain according to the geographical area: implications for genetic testing. Fam Cancer 2010; 9: 187-191.

14. Neuhausen SL, Godwin AK, Gershoni-Baruch R, et al. Haplotype and phenotype analysis of nine recurrent BRCA2 mutations in 111 families: results of an international study. Am J Hum Genet 1998; 62: 1381-1388.

15. van der Luijt RB, van Zon PHA, Jansen RPM, et al. De novo recurrent germline mutation of the BRCA2 gene in a patient with early onset breast cancer. J Med Genet 2001; 38: 102-105.

16. Rebbeck TR, Friebel TM, Friedman E, et al. Mutational spectrum in a worldwide study of 29,700 families with BRCA1 or BRCA2 mutations. Hum Mutat 2018; 39: 593-620.

17. Infante $M$, Durán $M$, Acedo A, et al. The highly prevalent BRCA2 mutation c.2808_2811del (3036delACAA) is located in a mutational hotspot and has multiple origins. Carcinogenesis 2013; 34: 2505-2511. 